Gynecologic and

Obstetric Investigation

\title{
Lack of an Association between a Polymorphism in the KRAS 3' Untranslated Region (rs61764370) and Endometriosis in a Large European Case-Control Study
}

\author{
Thomas M. D'Hooghe ${ }^{a}$ Olga Grechukhina ${ }^{b}$ SiHyun Cho $^{b}$ Amelie Fassbender ${ }^{a}$ \\ Dorien $\mathrm{O}^{\mathrm{a}}$ Daniëlle Peterse ${ }^{\mathrm{a}}$ Joanne Weidhaas ${ }^{c}$ Hugh S. Taylor ${ }^{\mathrm{a}}$ \\ ${ }^{a}$ Department of Development and Regeneration, Organ Systems, Catholic University (KU) Leuven, Leuven, Belgium; \\ ${ }^{b}$ Department of Obstetrics, Gynecology and Reproductive Sciences, Yale School of Medicine, New Haven, CT, USA; \\ 'Department of Radiation Oncology, David Geffen School of Medicine, University of California, Los Angeles, CA, USA
}

\section{Keywords}

Endometriosis $\cdot$ KRAS $\cdot$ Single-nucleotide polymorphism

\begin{abstract}
Background: Endometriosis is a common disorder that affects $6-10 \%$ of reproductive age women. In a previous study, we demonstrated that a polymorphism in let-7 microRNAbinding site in the $3^{\prime}$ untranslated region of the KRAS gene was found in $31 \%$ of subjects with endometriosis resistant to medical therapy. This polymorphism was now tested in a large, case-control study. Methods: Peripheral blood or peritoneal biopsies from 2,077 European subjects with or without endometriosis and known infertility were tested for the presence of the variant allele using polymerase chain reaction. Results: Histologically proven endometriosis was found in 1,140 subjects, while 937 subjects were disease free. Variant allele carrier rates in subjects with and without endometriosis were 15.7 and $15.1 \%$, respectively. No association between the variant KRAS allele and stage of the disease, age at surgery, body mass index, or type of infertility was identified. Conclusion: A germ-line single-nucleotide polymorphism in the let-7 microRNA-binding site of the KRAS gene was not associated with sporadic endometriosis in an infer-
\end{abstract}

\section{KARGER}

(c) 2019 S. Karger AG, Basel

E-Mail karger@karger.com

www.karger.com/goi tile Caucasian population in this large case-control study. However, it remains possible that this gene variant may be a marker of treatment resistance. Further studies on the role of this polymorphism in endometriosis are needed.

(c) 2019 S. Karger AG, Base

\section{Introduction}

Endometriosis is characterized by the presence of endometrial glands and stroma outside of endometrial cavity. The condition affects $6-10 \%$ of reproductive age women and is associated with chronic pelvic pain, dysmenorrhea, abnormal uterine bleeding, and infertility/ subfertility [1-3]. Endometriosis is found in up to $50 \%$ of patients with infertility $[1,4]$. Presentation of the disease may vary from nearly absent symptoms when the diagnosis is made incidentally during pelvic or abdominal surgery for other indications, to debilitating disease requiring multiple surgical interventions and associated with significant impairment of a woman's quality of life. Due

T.M.D. and O.G. contributed equally to the manuscript. 
to various and sometimes confusing clinical presentations, the diagnosis is commonly delayed by many years $[5,6]$.

Although multiple medications are being used for endometriosis-related symptoms (oral contraceptives, progestins, gonadotropin-releasing hormone agonists), medical therapy is frequently ineffective, whereas surgical management results in high recurrence of the disease (6-67\%), requiring repeat surgery in $36 \%$ of cases [7-10]. Such heterogeneity of presentations and discrepancies in treatment results may be related to disease variants, driven by distinct molecular phenotypes. A better understanding of these disease variants is needed to be able to individualize treatment and achieve better outcomes.

Although genetic predisposition to endometriosis has been proposed, specific genes responsible for endometriosis are yet to be identified [11]. In the only in vivo model of spontaneous murine endometriosis (Dinulescu et al. [12]), the activation of an oncogenic KRAS gene in the murine ovarian surface epithelium resulted in the de novo formation of lesions with endometriotic morphology and suggested that $R A S$ pathway activation may play a role in the development of endometriosis in humans. However, this observation has not been confirmed so far in women by mutational analysis of the coding regions of human KRAS gene [13-15].

MicroRNAs, small noncoding RNA molecules, are known to be powerful posttranscriptional regulators of gene expression [16]. They bind to the $3^{\prime}$ untranslated region (UTR) on the target messenger RNA (mRNA) and ultimately result in decreased protein translation. The let7 microRNA family is known to suppress translation of KRAS [17]. A specific single-nucleotide polymorphism (SNP) in the KRAS gene rs61764370, in which a singlenucleotide substitution $T$ (wild-type allele) $G$ (variant allele) occurs in a let-7 microRNA complementary site 6 (LCS6), was shown to be a risk factor for certain cancers, including nonsmall cell lung cancer [18], ovarian cancer in specific populations [19], and triple negative breast cancer [20]. Importantly, this polymorphism, now referred to as the KRAS-variant, was first suspected to be prognostic of poor outcome in cancer partly due to its enrichment in patients with metastatic disease [21-23]. However, it has now been definitively shown that instead, the KRAS-variant is a predictive biomarker of treatment response across many cancers, including ovarian cancer [24], head and neck cancer [22], colon cancer [25], and nonsmall cell lung cancer [26], with some standard of care treatments working well, and others poorly, allowing the development of advanced and recurrent disease.
Based on the extensive evidence for RAS pathway activation/alterations in endometriosis, we previously tested the hypothesis that KRAS-variant might be implicated in endometriosis [27]. In our study we found that 31\% (41 out of 132) of analyzed subjects with histologically proven endometriosis carried the KRAS-variant allele, a frequency much higher than that found in the general population $[18,27]$. Furthermore, in this study, endometriosis in women with the KRAS-variant was found to be biologically unique with higher proliferation rate and progesterone resistance. However, several studies by other groups failed to reveal an association between this variant and endometriosis [28]. In 2010, a genome-wide association (GWA) study in a Japanese population by Uno et al. [29] demonstrated a strong association between endometriosis and SNPs in CDKN2BAS (on chromosome 9p21) and on chromosome 1p36 which contained WNT4, but not with a SNP linked to the KRAS-variant rs61764370. Subsequently, Painter et al. [30] in 2011 identified association of an intergenic SNP on chromosome 7p15 with endometriosis in a large European cohort. In 2012, Luong et al. [31] analyzed GWA data that included 3,194 surgically confirmed endometriosis cases and 7,060 controls specifically for 2 SNPs that were known to be highly correlated with the rs61764370, followed by genotyping and concluded that there was no association between endometriosis and the KRAS-variant allele. Another GWAS by Albertsen et al. [32] demonstrated involvement of the area around WNT4 including 2 new genes HSPC157 and CDC42 in the development of endometriosis in European population. Using a Taqman assay, these authors were unable to confirm an association between the KRAS-variant allele rs61764370 and endometriosis in 1,123 cases and 832 controls.

In view of these conflicting results, we decided to assess the frequency of the variant KRAS-allele in patients with endometriosis in a large case-control study using the same method that was applied in our previous study. We thus sought to determine if the KRAS-variant allele is enriched in women with surgically documented endometriosis or, alternatively, instead might be a marker of treatment resistant endometriosis - a uniform characteristic of population included in our prior study.

\section{Materials and Methods}

Sample and Data Collection

A total of 2,077 women who had undergone a laparoscopy for subfertility with or without pain from January 1993 to February 2010 were included based on the following inclusion criteria: lapa-
D’Hooghe/Grechukhina/Cho/ Fassbender/O/Peterse/Weidhaas/Taylor 
roscopic and histologically proven presence of endometriosis or laparoscopic absence of endometriosis. All consecutive subjects undergoing surgery for subfertility were enrolled prospectively, and written informed consent was obtained from all women prior to the surgery. Peripheral blood samples or intraoperative peritoneal biopsies were collected. Women with endometriosis had either minimal (stage I), mild (stage II), moderate (stage III), or severe (stage IV) disease, staged according to the revised American Fertility Society (AFS) classification system [33]. In cases, where endometriosis was diagnosed, surgical resection or ablation was performed. The study was approved by the Commission for Medical Ethics of the Leuven University Hospital, Belgium, and the Regional Ethics Committee, Karolinska Institute, Stockholm, Sweden.

\section{DNA Extraction}

As previously described (Sundqvist et al. [34]), DNA purified from EDTA-stabilized whole peripheral blood was collected for routine molecular diagnostic tests at the Centre for Human Genetics of University Hospitals, Leuven, Belgium. Various purification methods were used: Chemagic DNA blood special kit (Chemagen MSM I, PerkinElmer Chemagen Technologies GmbH, Baesweiler, Germany), Auto Pure LS Puregene chemistry (Qiagen, Venlo, The Netherlands). The choice of the extraction method was based on the available amount of blood and on the type of required molecular diagnostic test. DNA from peritoneal biopsies was purified with QIAGEN QIAamp DNA mini kit (QIAGEN, Hilden, Germany) according to the manufacturer's protocol. DNA concentration was measured with NanoDrop 1,000 (Thermo Scientific, Wilmington, DE, USA). For tissue samples with 260:280 ratios <1.5, a DNA clean-up/purification with phenol: chloroform (P 2069, Sigma-Aldrich, Saint Louis, MO, USA) was performed. DNA concentration was measured using Victor (PerkinElmer, MA, USA).

\section{Genetic Testing}

For high-throughput genotyping, the isolated DNA samples were amplified using TaqMan polymerase chain reaction assays designed specifically to identify the T or G allele of the LCS6 SNP of KRAS gene (Applied Biosystems, Foster City, CA, USA) as was described previously [18]. The work was performed at MiraDx, in their CLIA approved laboratory, using their KRAS-variant allele assay.

\section{Statistical Analysis}

Statistical analysis of the data was performed using IBM SPSS Statistical Data Editor. Chi-square test was used to analyze categorical data (frequencies of different alleles, types/causes of infertility, stages of endometriosis), while student $t$ test and 1-way ANOVA were used to compare interval data (body mass index [BMI], ages, AFS scores). Statistical significance was determined as $p<0.05$.

\section{Results}

\section{Group Characteristics}

A total of 2,077 subjects were analyzed for the KRASvariant, all of which underwent laparoscopic surgery for infertility or subfertility. About $90 \%$ of subjects included in the study were European Caucasians. For most patients, this was their initial surgery indicated by infertility/subfertility. Totally, 1,140 women had histologically proven endometriosis, while 937 had no laparoscopic evidence of the disease. Infertility was documented in the medical records of $1,071 / 1,140$ and in $889 / 937$ in the groups with and without endometriosis, respectively. Subjects were noted to be of older age at the time of the surgery in the group without endometriosis (31.4 vs. 32.1 year old, $p=0.0036$; Table 1 ). Subjects with endometriosis were more likely to have primary rather than secondary infertility compared to those without endometriosis (76 vs. $62 \%, p=0.0001$ ). Subjects without endometriosis were more likely to be smokers ( 14.4 vs. $10.7 \%, p=0.011$ ). In cases where the cause of infertility/subfertility was known, the groups with endometriosis versus no endometriosis were statistically different based on this stratification. Ovulation, implantation, tubal, and genetic problems were identified in $9.5,1.4,5.6$, and $0.3 \%$, respectively, in the group with a known endometriosis and 20.2, 3.5, 24.9 , and $1 \%$ in the group without laparoscopic evidence of endometriosis $(p<0.00001)$. In $83 \%$ of cases with endometriosis, the cause of infertility was categorized as "unexplained" versus $50 \%$ of such cases in the group without endometriosis (Table 1). "Unexplained" infertility in patients with endometriosis was frequently ascribed to endometriosis itself, however, still classified as unexplained. In the group with endometriosis, the stage of the disease stratification was as follows: Stage I - 34.4\%, Stage II - 21.3\%, Stage III - 16.5\%, and Stage IV - 26.5\%. About $1.1 \%$ of patients with endometriosis did not have an assigned stage in their chart. Mean AFS score for all endometriosis patients was $29 \pm 33$.6. Subjects who smoke daily were more likely to have tubal factor infertility than non-smokers.

\section{Frequency of $G$ Versus T Alleles in LCS6 on 3'UTR of the KRAS Gene}

In the entire tested population ( $n=2,077)$, the TT genotype (aka wild type) was found in $84.5 \%(n=1,756)$, TG in $14.8 \%(n=308)$, and GG in $0.6 \%(n=13)$ of cases. The frequency of the $\mathrm{G}$ allele (variant) was thus calculated as 8.04\% ([number of G alleles in heterozygotes + number of $\mathrm{G}$ alleles in homozygotes $\times 2$ ]/total number of $\mathrm{T}$ and $\mathrm{G}$ alleles $=[308+13 \times 2] / 2,077 \times 2)$. Due to a very low frequency of cases homozygous for the $\mathrm{G}$ allele $(n=13)$, we considered heterozygous cases with TG genotype to be positive for a variant allele and cases homozygous for $G$ allele (GG genotype) were added to this group in all subsequent 
Table 1. Comparison of the groups with and without endometriosis

\begin{tabular}{lccc}
\hline & Endometriosis & No endometriosis & $p$ value \\
\hline Number & 1,140 & 937 & 0.001 \\
BMI, mean (SD) & $23.1(4.19)$ & $23.9(4.53)$ & 0.39 \\
Infertility - any, $n(\%)$ & $1,071(93.9)$ & $889(94.8)$ & 0.0001 \\
Primary, $n$ (\%) & $822(72.1)$ & $556(59.3)$ & 0.0001 \\
Secondary, $n$ (\%) & $249(21.8)$ & $333(35.5)$ & 0.00001 \\
$\quad$ Anovulation & $102(9.5)$ & $180(20.2)$ & 0.00001 \\
$\quad 15(1.4)$ & $222(24.9)$ & $9(1)$ \\
$\quad$ Implantation & $60(5.6)$ & $446(50)$ & $136(14.5)$ \\
$\quad$ Genetic & $4(0.3)$ & $32.1(4.8)$ & 0.0112 \\
$\quad$ Unexplained & $123(10.7)$ & 0.0036 \\
Smoking, $n$ (\%) & $31.4(4.3)$ & & \\
\hline
\end{tabular}

BMI, body mass index.

Table 2. Genotype distribution in patients with and without endometriosis

\begin{tabular}{lllllllll}
\hline Genotype & \multicolumn{2}{l}{ Endometriosis } & & & \multicolumn{2}{l}{ No endometriosis } \\
\cline { 2 - 3 } & TT & TG & GG & & TT & TG & GG \\
\hline$n(\%)$ & $961(84.2)$ & $177(15.5)$ & $2(0.17)$ & & $795(84.8)$ & $133(14.2)$ & $11(1.1)$ \\
G allele frequency, $\%$ & & & 7.90 & & 8.20 & \\
\hline
\end{tabular}

analyses. The prevalence of women who were positive for a variant allele was similar ( $p=0.81$; Table 2$)$ in the endometriosis group (15.7\%; G allele frequency $7.9 \%$ ) and in the control group (15.1\%; $G$ allele frequency $8.2 \%)$. In the previous study variant allele frequency was $18.1 \%$ in endometriosis group compared to $7.6 \%$ variant allele frequency in the general mixed European population. In order to detect the same difference between the control and endometriosis groups, maintaining a power of $80 \%$ with an alpha-I error of 0.05 , we would need to test a total of only 316 alleles (158 alleles in each group). In this study, a total of 4,154 alleles were tested, providing sufficient power to detect not only the same difference which we saw in the previous paper but also a difference as small as $2.6 \%$.

\section{Comparison of Patients with and without \\ Endometriosis Carrying Wild Type and Variant \\ KRAS Alleles}

The frequency of primary versus secondary infertility in patients with and without endometriosis did not differ depending on the presence of wild type or variant alleles. In cases where the cause of infertility was known (ovulation, implantation, tubal, or genetic), variant allele was not associated with any individual etiology (Table 3). Patients in all 4 groups had the same BMI and age distribution at the time of surgery. Endometriosis subjects carrying wild type and variant KRAS alleles were not statistically different with respect to their AFS score (30.4 vs. 26.1 in the groups carrying variant vs. wild type alleles, $p=0.17$ ) and thus the stage of endometriosis (Table 4). One of the 2 endometriosis patients homozygous for variant allele had an AFS score of 112, while the other did not have an AFS score assigned. Both cases were excluded from the AFS score analysis.

The variant allele did not have any association with $\mathrm{BMI}$, age at surgery, or type of infertility in patients from both groups (with and without endometriosis) when stratification by the presence of endometriosis was removed (Table 5).

\section{Discussion}

In our previous study, we found that $31 \%$ of patients with moderate to severe endometriosis (undergoing surgery for treatment of pain and failure of medical therapy; 
Table 3. Genotype distribution in groups with and without endometriosis stratified by type and causes of infertility

\begin{tabular}{|c|c|c|c|c|c|}
\hline & \multicolumn{2}{|c|}{ Endometriosis, $n(\%)$} & \multicolumn{2}{|c|}{ No endometriosis, $n(\%)$} & \multirow[t]{2}{*}{$p$ value } \\
\hline & WT & $\mathrm{KV}$ & WT & KV & \\
\hline Number & 961 & 179 & 795 & 142 & 0.81 \\
\hline Infertility & $905(94.1)$ & $166(92.7)$ & $752(94.5)$ & $137(96.4)$ & 0.55 \\
\hline Primary & $693(72.1)$ & $129(72)$ & $476(59.8)$ & $80(56.3)$ & 0.59 \\
\hline Secondary & $212(22)$ & $37(21.5)$ & $276(34.7)$ & $57(40.1)$ & 0.42 \\
\hline Ovulation & $89(9.2)$ & $13(7.2)$ & $152(19.1)$ & $28(19.7$ & 0.4 \\
\hline Implantation & $12(1.2)$ & $3(1.6)$ & $29(3.6)$ & $3(2.1)$ & 0.3 \\
\hline Transport & $49(5)$ & $11(6.1)$ & $197(24.5)$ & $25(14.7)$ & 0.14 \\
\hline Genetics & $3(0.31)$ & $1(0.5)$ & $8(1)$ & $1(7)$ & 0.5 \\
\hline Unexplained & $752(78.2)$ & $138(77.6)$ & $366(46)$ & $80(56.3)$ & 0.25 \\
\hline
\end{tabular}

WT, wild type, TT allele carriers; KV, KRAS variant, TG or GG allele carriers.

Table 4. Frequency of wild-type and variant alleles in different stages of endometriosis

\begin{tabular}{llcc}
\hline & \multicolumn{2}{l}{ Endometriosis, $n(\%)$} & \multirow{2}{*}{$p$ value } \\
\cline { 2 - 3 } & WT & KV & \\
\hline Stage & & & \\
I & $323(34)$ & $70(39.1)$ & \\
II & $205(21.3)$ & $38(21.2)$ & \\
III & $159(16.5)$ & $29(16.2)$ & \\
IV & $262(27.2)$ & $41(22.9)$ & \\
Unknown & $12(1.2)$ & $2(1.1)$ & \\
\hline
\end{tabular}

WT, wild type; KV, KRAS variant.

$n=132 ; \sim 90 \%$ with stage III-IV endometriosis) were carrying a polymorphism (T-G) in the let-7 complementary site 6 in the 3'UTR of the KRAS gene. We then proposed that this polymorphism may be associated with severe forms of this disease and possibly play a role in its development. However, further large-scale GWA studies failed to demonstrate increased frequency of the variant allele in patients with endometriosis. In this study, we used a much larger patient population from Europe to analyze the frequency of this polymorphism in a case-control study. We used the same direct method of identifying the polymorphism via TaqMan polymerase chain reaction, which was used in our original study. We found the variant allele frequency to be $\sim 8 \%$, which is similar to the previously described frequency of this allele in European population [18]. We did not demonstrate an increased frequency of a variant allele in the patients with endometriosis, nor were we able to identify any association be-

KRAS Polymorphism in Endometriosis Patients. tween the variant allele and other clinical characteristics that were analyzed. We did not see an association between the variant allele and more severe forms of endometriosis. Unfortunately, in this study, we were not able to analyze the association of the KRAS-variant with other clinical features of endometriosis such as pain and abnormal uterine bleeding, since this was an infertility cohort and these clinical data had not been documented consistently in the patient files. In our original paper, we did find the polymorphism to be associated with infertility but not with chronic pelvic pain, dysmenorrhea and dyspareunia.

There may be several explanations for the discrepancy of the results of this study and our previous data. The method of identifying the polymorphism was identical in both studies, which minimizes the possibility of procedural differences and errors. All subjects included in this study were diagnosed with infertility, which could have been a confounding factor. All subjects included in our previous study were referred to a university reproductive endocrinology and infertility clinic specializing in endometriosis from community providers, which indicates that the subjects in our former study had undergone and failed multiple therapies and had prior repeated surgeries for endometriosis. In the current study, the subjects were seen for the evaluation of infertility and not selected based on failure of prior therapy. In fact, it is likely than many had no prior therapy. The high incidence of the variant allele in the subjects from our previous study may be a marker of resistance to medical and possibly surgical therapy or nonresponsiveness to infertility treatment rather than an indicator of predisposition to it. Although we did not see enrichment in Stage IV patients in the cur- 
Table 5. Comparison of BMI, age at surgery, and types of infertility in patients carrying wild-type or variant alleles

\begin{tabular}{lrrl}
\hline & \multicolumn{1}{l}{ KV } & WT & $p$ value \\
\hline BMI, mean (SD) & $23.5(4.33)$ & $23.46(4.34)$ & 0.9 \\
Age at surgery, mean (SD) & $32.07(4.55)$ & $31.65(4.54)$ & 0.18 \\
Infertility $(n=1,960), n(\%)$ & $303(15.4)$ & $1,657(84.5)$ & \\
$\quad$ Primary $(n=1,378)$ & $209(15.1)$ & $1,169(84.9)$ & 0.581 \\
Secondary $(n=582)$ & $94(16.1)$ & $488(83.8)$ & \\
\hline
\end{tabular}

BMI, body mass index; WT, wild type; KV, KRAS variant.

rent study, the high prevalence of KRAS-variant patients in our previous cohort of extensively treated patients who had failed standard treatment suggests that $K R A S$-variant patients do have treatment resistant, aggressive endometriosis.

Examples of the existence of subsets of patients who respond differently to standard therapy can be found extensively in cancer. One of the first examples is ER + breast cancer, which comprises up to $2 / 3$ of breast cancer patients. Tamoxifen was one of the first "personalized medicines" applied to better treat these patients. Another example is HER-2/neu oncogene overexpression that is found in approximately $15 \%$ of breast cancer patients. While patients with HER-2/neu overexpressing tumors were originally found to have poor outcomes, by using this information to deliver trastuzumab (Herceptin) for the past 10 years, this biomarker is now considered an example of ideal personalized medicine [35]. The KRAS-variant is proving to be a strong predictive biomarker in cancer treatment as well, with $K R A S$-variant patients exhibiting cisplatin resistance in ovarian and head and neck cancer $[22,24]$ and cetuximab sensitivity in colon cancer [25] and head and neck cancer [36]. Thus, the hypothesis that the KRAS-variant could be a predictive biomarker of treatment response in endometriosis is not only plausible, but likely. Our previous finding of decreased progesterone receptor expression, increased endometrial cell proliferation rates, and enrichment of KRAS-variant patients in high risk treatment resistant endometriosis patients strongly supports the hypothesis that subjects carrying the variant allele may identify a subgroup of endometriosis patients who will have a poor response to standard therapies [27]. This hypothesis deserves further evaluation.

While this study was much better powered and indicates that the KRAS-variant rs61764370 is not a marker of susceptibility to endometriosis in European populations, there are still many questions about the role of the $K R A S$-variant in the pathophysiology of endometriosis. In our original paper, we demonstrated that KRAS mRNA expression was increased in eutopic endometrium of subjects with endometriosis compared to endometrium of healthy subjects [27]. KRAS overexpression was associated with downregulation of the let-7 microRNA family in endometrium from subjects with endometriosis, with let-7b and let-7e being significantly decreased, confirming the known feedback loop between let-7 and KRAS. Similarly, Cho et al. [37] in 2015 demonstrated decreased circulating let-7 microRNA levels in the serum of subjects with endometriosis. Several other groups also demonstrated elevated KRAS mRNA levels in eutopic endometrium of subjects with endometriosis in both human and baboon studies [38-40]. Another group (Laudanski et al. [41]) did not see statistically different expression of KRAS mRNA in eutopic endometrium from subjects with and without endometriosis. However, in their study, the levels of KRAS mRNA were significantly higher in ovarian endometriosis compared to eutopic endometrium [41]. With more emerging data on KRAS and let-7 expression in subjects with endometriosis (eutopic vs. ectopic endometrium in subjects with endometriosis, eutopic endometrium from subjects with and without endometriosis, serum from subjects with and without endometriosis), it is becoming clear that this interaction plays a meaningful role in the disease; however, the direct mechanism, molecular pathway, effects, and clinical significance of this interaction require more investigation.

In conclusion, a germ-line SNP in the let-7 microRNAbinding site of the KRAS gene was not associated with endometriosis in Caucasian population in a large case-control study; however, it may be a marker of resistance to standard therapies and aggressive disease. 
Limitations and Strengths of the Study

Limitations of the study include the retrospective design and that only women with infertility were included. Women with severe pain syndrome, which oftentimes would represent treatment-resistant group, were not included in the study. Also, this was a fairly homogenous population of Caucasian women of European descent, while in our original study, and there was a substantial number of Hispanic and African-American women. The use of a standardized genotyping as well as laparoscopic confirmation of the presence of absence of the disease in a large population is a significant strength of the study.

\section{References}

1 Giudice LC. Clinical practice. Endometriosis. N Engl J Med. 2010 Jun;362(25):238998.

2 Giudice LC, Kao LC. Endometriosis. Lancet. 2004 Nov;364(9447):1789-99.

3 Eskenazi B, Warner ML. Epidemiology of endometriosis. Obstet Gynecol Clin North Am. 1997 Jun;24(2):235-58.

4 Macer ML, Taylor HS. Endometriosis and infertility: a review of the pathogenesis and treatment of endometriosis-associated infertility. Obstet Gynecol Clin North Am. 2012 Dec;39(4):535-49.

5 Hudelist G, Fritzer N, Thomas A, Niehues C, Oppelt P, Haas D, et al. Diagnostic delay for endometriosis in Austria and Germany: causes and possible consequences. Hum Reprod. 2012 Dec;27(12):3412-6.

6 Brosens I, Gordts S, Benagiano G. Endometriosis in adolescents is a hidden, progressive and severe disease that deserves attention, not just compassion. Hum Reprod. 2013 Aug; 28(8):2026-31.

7 Abbott JA, Hawe J, Clayton RD, Garry R. The effects and effectiveness of laparoscopic excision of endometriosis: a prospective study with 2-5 year follow-up. Hum Reprod. 2003 Sep;18(9):1922-7.

8 Guo SW. Recurrence of endometriosis and its control. Hum Reprod Update. 2009 Jul-Aug; 15(4):441-61.

9 Dunselman GA, Vermeulen N, Becker C, Calhaz-Jorge C, D'Hooghe T, De Bie B, et al.; European Society of Human Reproduction and Embryology. ESHRE guideline: management of women with endometriosis. Hum Reprod. 2014 Mar;29(3):400-12.

10 Taylor HS, Giudice LC, Lessey BA, Abrao MS, Kotarski J, Archer DF, et al. Treatment of Endometriosis-Associated Pain with Elagolix, an Oral GnRH Antagonist. N Engl J Med. 2017 Jul;377(1):28-40.

11 Moen MH, Magnus P. The familial risk of endometriosis. Acta Obstet Gynecol Scand. 1993 Oct;72(7):560-4.

12 Dinulescu DM, Ince TA, Quade BJ, Shafer SA, Crowley D, Jacks T. Role of K-ras and Pten in the development of mouse models of endometriosis and endometrioid ovarian cancer. Nat Med. 2005 Jan;11(1):63-70.

13 Zhao ZZ, Nyholt DR, Le L, Martin NG, James MR, Treloar SA, et al. KRAS variation and risk of endometriosis. Mol Hum Reprod. 2006 Nov;12(11):671-6.
14 Vestergaard AL, Thorup K, Knudsen UB, Munk T, Rosbach H, Poulsen JB, et al. Oncogenic events associated with endometrial and ovarian cancers are rare in endometriosis. Mol Hum Reprod. 2011 Dec;17(12):758-61.

15 Li X, Zhang Y, Zhao L, Wang L, Wu Z, Mei Q, et al. Whole-exome sequencing of endometriosis identifies frequent alterations in genes involved in cell adhesion and chromatin-remodeling complexes. Hum Mol Genet. 2014 Nov;23(22):6008-21.

16 Grosshans H, Slack FJ. Micro-RNAs: small is plentiful. J Cell Biol. 2002 Jan;156(1):17-21.

17 Johnson SM, Grosshans H, Shingara J, Byrom $\mathrm{M}$, Jarvis R, Cheng A, et al. RAS is regulated by the let-7 microRNA family. Cell. 2005 Mar; 120(5):635-47.

18 Chin LJ, Ratner E, Leng S, Zhai R, Nallur S, Babar I, et al. A SNP in a let-7 microRNA complementary site in the KRAS $3^{\prime}$ untranslated region increases non-small cell lung cancer risk. Cancer Res. 2008 Oct;68(20): 8535-40.

19 Ratner E, Lu L, Boeke M, Barnett R, Nallur S, Chin LJ, et al. A KRAS-variant in ovarian cancer acts as a genetic marker of cancer risk. Cancer Res. 2010 Aug;70(16):6509-15.

20 Paranjape T, Heneghan H, Lindner R, Keane FK, Hoffman A, Hollestelle A, et al. A 3'-untranslated region KRAS variant and triplenegative breast cancer: a case-control and genetic analysis. Lancet Oncol. 2011 Apr;12(4): $377-86$.

21 Christensen BC, Moyer BJ, Avissar M, Ouellet LG, Plaza SL, McClean MD, et al. A let-7 microRNA-binding site polymorphism in the KRAS $3^{\prime}$ UTR is associated with reduced survival in oral cancers. Carcinogenesis. 2009 Jun;30(6):1003-7.

22 Chung CH, Lee JW, Slebos RJ, Howard JD, Perez J, Kang H, et al. A 3'-UTR KRAS-variant is associated with cisplatin resistance in patients with recurrent and/or metastatic head and neck squamous cell carcinoma. Ann Oncol. 2014 Nov;25(11):2230-6

23 Graziano F, Canestrari E, Loupakis F, Ruzzo A, Galluccio N, Santini D, et al. Genetic modulation of the Let-7 microRNA binding to KRAS $3^{\prime}$-untranslated region and survival of metastatic colorectal cancer patients treated with salvage cetuximab-irinotecan. Pharmacogenomics J. 2010 Oct;10(5):458-64.

24 Ratner ES, Keane FK, Lindner R, Tassi RA, Paranjape T, Glasgow M, et al. A KRAS vari- ant is a biomarker of poor outcome, platinum chemotherapy resistance and a potential target for therapy in ovarian cancer. Oncogene. 2012 Oct;31(42):4559-66.

25 Saridaki Z, Weidhaas JB, Lenz HJ, LaurentPuig P, Jacobs B, De Schutter J, et al. A let-7 microRNA-binding site polymorphism in KRAS predicts improved outcome in patients with metastatic colorectal cancer treated with salvage cetuximab/panitumumab monotherapy. Clin Cancer Res. 2014 Sep;20(17):4499510.

26 Weidhaas J, Kim E, Herbst R, Yu J, Slack F, Blumenschein G, et al. The KRAS-variant and treatment response in BATTLE-1. J Clin Oncol 2014;32:suppl:abstr 8135.

27 Grechukhina O, Petracco R, Popkhadze S, Massasa E, Paranjape T, Chan E, et al. A polymorphism in a let-7 microRNA binding site of KRAS in women with endometriosis. EMBO Mol Med. 2012 Mar;4(3): 206-17.

28 Rahmioglu N, Nyholt DR, Morris AP, Missmer SA, Montgomery GW, Zondervan KT. Genetic variants underlying risk of endometriosis: insights from meta-analysis of eight genome-wide association and replication datasets. Hum Reprod Update. 2014 Sep-Oct; 20(5):702-16.

29 Uno S, Zembutsu H, Hirasawa A, Takahashi A, Kubo M, Akahane T, et al. A genome-wide association study identifies genetic variants in the CDKN2BAS locus associated with endometriosis in Japanese. Nat Genet. 2010 Aug; 42(8):707-10.

30 Painter JN, Anderson CA, Nyholt DR, Macgregor S, Lin J, Lee SH, et al. Genome-wide association study identifies a locus at $7 \mathrm{p} 15.2$ associated with endometriosis. Nat Genet. 2011 Jan;43(1):51-4.

31 Luong HT, Nyholt DR, Painter JN, Chapman B, Kennedy S, Treloar SA, et al. No evidence for genetic association with the let-7 microRNA-binding site or other common KRAS variants in risk of endometriosis. Hum Reprod. 2012 Dec;27(12):3616-21.

32 Albertsen HM, Chettier R, Farrington P, Ward K. Genome-wide association study link novel loci to endometriosis. PLoS One. 2013; 8(3):e58257.

33 ASRM. Revised American Society for Reproductive Medicine classification of endometriosis: 1996. Fertil Steril. 1997 May;67(5):81721. 
34 Sundqvist J, Falconer H, Seddighzadeh M, Vodolazkaia A, Fassbender A, Kyama C, et al. Ovarian cancer-associated polymorphisms in the BNC2 gene among women with endometriosis. Hum Reprod. 2011 Aug;26(8):2253-7.

35 Ross JS, Slodkowska EA, Symmans WF, Pusztai L, Ravdin PM, Hortobagyi GN. The HER2 receptor and breast cancer: ten years of targeted anti-HER-2 therapy and personalized medicine. Oncologist. 2009 Apr;14(4):32068.

36 Weidhaas JB, Harris J, Schaue D, Chen AM, Chin R, Axelrod R, et al. The KRAS-Variant and Cetuximab Response in Head and Neck Squamous Cell Cancer: A Secondary Analysis of a Randomized Clinical Trial. JAMA Oncol. 2017 Apr;3(4):483-91.

37 Cho S, Mutlu L, Grechukhina O, Taylor HS. Circulating microRNAs as potential biomarkers for endometriosis. Fertil Steril. 2015 May;103(5):1252-60.e1.

38 Farahani MS, Shahbazi S, Moghaddam SA, Mahdian R. Evaluation of KRAS Gene Expression and LCS6 Variant in Genomic and Cell-Free DNA of Iranian Women With Endometriosis. Reprod Sci. 2015 Jun;22(6):67984.

39 Shahrabi-Farahani M, Shahbazi S, Mahdian R, Amini-Moghaddam S. K-Ras 4A Transcript variant is up-regulated in eutopic endo- metrium of endometriosis patients during proliferative phase of menstrual cycle. Arch Gynecol Obstet. 2015 Jul;292(1):225-9.

40 Afshar Y, Hastings J, Roqueiro D, Jeong JW, Giudice LC, Fazleabas AT. Changes in eutopic endometrial gene expression during the progression of experimental endometriosis in the baboon, Papio anubis. Biol Reprod. 2013 Feb;88(2):44.

41 Laudanski P, Szamatowicz J, Kowalczuk O, Kuźmicki M, Grabowicz M, Chyczewski L. Expression of selected tumor suppressor and oncogenes in endometrium of women with endometriosis. Hum Reprod. 2009 Aug; 24(8):1880-90.
D'Hooghe/Grechukhina/Cho/ Fassbender/O/Peterse/Weidhaas/Taylor 